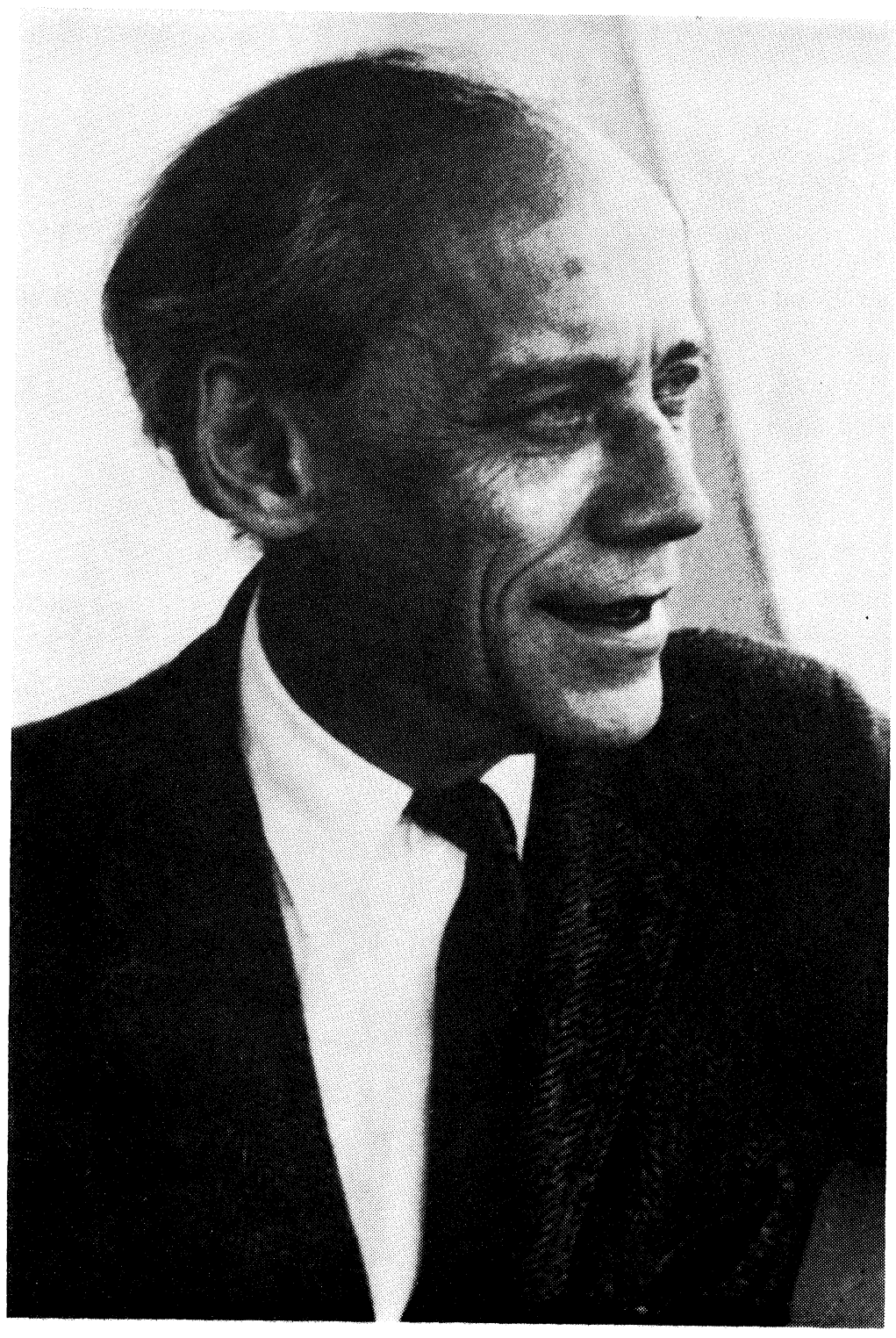





\section{EMIL ARTIN}

\section{BY RICHARD BRAUER}

Emil Artin died of a heart attack on December 20, 1962 at the age of 64. His unexpected death came as a tremendous shock to all who knew him. There had not been any danger signals. It was hard to realize that a person of such strong vitality was gone, that such a great mind had been extinguished by a physical failure of the body.

Artin was born in Vienna on March 3, 1898. He grew up in Reichenberg, now Tschechoslovakia, then still part of the Austrian empire. His childhood seems to have been lonely. Among the happiest periods was a school year which he spent in France. What he liked best to remember was his enveloping interest in chemistry during his high school days. In his own view, his inclination towards mathematics did not show before his sixteenth year, while earlier no trace of mathematical aptitude had been apparent. ${ }^{1} \mathrm{I}$ have often wondered what kind of experience it must have been for a high school teacher to have a student such as Artin in his class.

During the first world war, he was drafted into the Austrian Army. After the war, he studied at the University of Leipzig from which he received his Ph.D. in 1921. He became "Privatdozent" at the University of Hamburg in 1923. This is the first academic position in German Universities; the "venia legendi" that goes with it is the right to hold classes.

Now there began a period of the strongest and most fruitful mathematical activity for Artin. He rose quickly in the academic ranks becoming a full professor in 1926. Hamburg, then only a few years old and the youngest German University, had already become one of the leading ones. Its special role in mathematics can be seen, if one looks through the volumes of the "Abhandlungen aus dem Mathematischen Seminar Hamburg." Of course, Artin himself had a great share in the rise to prominence of this journal.

The intellectual atmosphere of German universities of that period is remembered with nostalgia by all who knew it. Artin, with his wide interests in all fields of human endeavor became the stimulating center of a circle of friends. His strange nickname " $\mathrm{Ma}$ " which he always preferred to his given name Emil goes back to those days. It is short for "Mathematics"; he simply appeared to these young men as an embodiment of mathematics.

1 In [49], he says: "Meine eigene Vorliebe zur Mathematik zeigte sich erst im sechzehnten Lebensjahr, während vorher von irgendeiner Anlage dazu überhaupt nicht die Rede sein konnte." 
In 1929, Artin married a student of his, Natalie Jasny who has become known as Natasha among mathematicians. Through her warm personality and helpfulness, her many talents, and her charm, Natasha in her own right has acquired a unique position in the international mathematical community.

Artin never did anything by halves. His family now occupied a central position in his life. When his children were growing up, he took a most active part in all phases of their education. He spent hours with them every day, and it was of foremost importance to him to instill in them his own personal and cultural standards. ${ }^{2}$

In 1933, Hitler and the Nazi party came to power in Germany. It was only a question of time until Artin, with his feeling for individual freedom, his sense of justice, his abhorrence of physical violence, would leave Germany. In 1937, he and his family emigrated to America. After a year at the University of Notre Dame, he became Professor at Indiana University. At once, he began to make his ideas on teaching felt, and he assembled around him an active mathematical group. Although he had become very much attached to Bloomington, he moved to Princeton in 1946. For Artin, for whom his teaching was of the utmost importance, Princeton appeared as the place where his pedagogical activities would be most fruitful. This has come true even beyond expectations.

In 1956 Artin took sabbatical leave, the first of his life. Before that time he had avoided visiting Germany again. He now accepted an invitation to go to the University of Göttingen as Gauss Professor for a term, and to teach another term at the University of Hamburg. Both in Göttingen and Hamburg, he had friends and personal ties. $\mathrm{He}$ had spent a year in Göttingen as a young Ph.D. It was the place where Gauss, Riemann, Dirichlet, Hilbert, Minkowski and so many other great mathematicians had lived and worked. In Hamburg, he himself had taken part in the building of a new great university. His years there had been the most productive time of his life.

During this year in Germany, he came to the decision to return there on a permanent basis. He taught at Princeton for one more year. In 1958, he accepted a position as Professor at the University of Hamburg, and he spent the last four years of his life in Hamburg.

I saw Artin for the last time in November 1958 in Hamburg. He spoke with satisfaction of his life and work in the United States. In Princeton, John Tate and Serge Lang had been his students. "This

2 Karin Artin is now the wife of John Tate, Michael Artin is Professor of Mathematics at the Massachusetts Institute of Technology. Thomas Artin is an instructor in the English Department of Swarthmore College. 
happens only once to a man. Not many mathematicians have been that lucky" were his words. He was content with his new life. There were vague plans of visits to America, but it was clear that Hamburg was to remain his home.

We took a long walk one afternoon talking of old times. It was one of those misty, melancholy, and rather miserable days which all northern harbor cities know so well in late fall. We wandered endlessly through the streets searching, I did not know for what, until I realized, it was a Hamburg which no longer existed and times which were gone for ever. Before Artin's eyes, I believe, there must have been the picture of the young Artin who had walked through the same streets thirty years before, full of life and strength.

The reproduction of the photograph will give an idea of Artin's appearance. It cannot show the very startling blue of his eyes. He looked exactly as one would have imagined that he should look. People who knew about him without knowing him by sight could often identify him in a crowd.

I shall now try to describe various aspects of Artin's personality. Artin was as much an artist as he was a scientist. His love of music was perhaps as deep as his love of mathematics. There seemed to be a great deal of the mathematician in Artin, the musician, and a great deal of the artist in Artin, the mathematician. In talking about the future development of mathematical theories, he could sometimes resemble the pianist sitting at the keyboard and following his phantasies. Some ideas stood out in clear detail. Others were still obscured and connections were missing. Eventually, one felt, a whole symphony would evolve. From the very beginning, there was always a great vision.

We like to think that every truly great mathematician is also a great human being and that there is something unique, not only in his mathematics, but in his whole nature. This may not always be true, but it was certainly true for Artin. The symbiosis of the scientist and the artist in Artin was unique.

If Artin had not become a mathematician, would he have distinguished himself in whatever field he had chosen? There cannot be any doubt about it. There were many subjects on which he had an amazingly deep knowledge for a layman, Chemistry, Astronomy, History of Music, and others. He was once questioned by some biochemists after his brilliant Sigma Xi lecture on "braids." They wanted to know what possible effect such abstract speculations could have 
for the good of humanity. He answered with a discourse on theoretical work in biochemistry discussing details which at first seemed to have been lacking in practical value and later had turned out to be of high importance. When building a telescope as a hobby, he studied the refined techniques used in the construction of some of the most modern instruments. To turn in a different direction: When he visited Japan at the time of the Nikko Symposium on Algebraic Numbers, he displayed great interest in Buddhism. In order to be able to answer some of his questions, our Japanese colleagues had to consult outstanding experts. At some time thirty years before, Artin had read widely on the subject.

Whatever Artin did, he did with full concentration and, one might say, singlemindedness. In spite of his wide interests, his creative work was all in mathematics. Mathematics was the natural instrument for his particular kind of intelligence, the field in which his special power of reasoning would find its purest application. It was his belief that the same kind of reasoning had its place in all sciences, and if mathematics needed justification, this was it. But we don't justify music, and why should we justify mathematics?

Artin was a man of varying moods. He could enjoy life fully. There were many things he liked, new impressions, a good conversation, good food. During his Bloomington years, his students would gather in his home after seminars, and he was relaxed. His teaching could be a great source of satisfaction.

He was never aloof. He was a man with an instinctive understanding for the feelings of other people, a person on whom you could rely, a warm friend.

We should now look at Artin's mathematical work. Since class field theory was the field closest to his heart, we begin with this theory.

During the years Artin was a student, Takagi's fundamental papers had appeared. They represented the crowning effort of a long development which incorporated some of the most vital parts of number theory. Without trying to convey an idea of its importance, we shall describe it briefly and rather vaguely.

Let $k$ be a fixed algebraic number field (of finite degree over the field $Q$ of rational numbers). We say that a normal extension field $K$ is abelian, if the Galois group $G(K / k)$ is abelian. With each such abelian extension field $K$, Takagi associates a certain object $T(K)$ defined in $k$. Actually, $T(K)$ is a class of ideal class groups $\mathfrak{h} / \mathfrak{h}_{0}$ of $k$, equivalent under an equivalence relation. The set of all these $T(K)$ can be described and each $T(K)$ characterizes an abelian extension 
field $K$ of $k$ uniquely. On the other hand, the most important algebraic and arithmetical properties of $K$ can be obtained immediately from $T(K)$. The Galois group $G(K / k)$ is isomorphic to the groups $\mathfrak{h} / \mathfrak{h}_{0}$ in $T(K)$. If $\mathfrak{p}$ is a prime ideal of $k$, the form of its decomposition into prime ideals of $K$ is determined by $T(K)$. Takagi's proofs were extremely long and difficult. Analytic arguments were needed in which generalizations of Dirichlet's $L$-series played an important role.

At once the following question comes to one's mind. Can one develop a similar theory in which not only abelian extension fields of $K$ but all algebraic extension fields $K$ of $k$ are considered? We wish then to describe explicitly a set of objects $T$ determined by the given field $k$, such that these $T$ are in one-to-one correspondence to the extension fields $K$, and that from each $T$, the algebraic and arithmetical properties of the corresponding $K$ can be read off. This is what we have in mind when we speak of a "nonabelian" class field theory. We are still in the dark today how such a theory will have to look. We cannot today describe explicitly the lattice of algebraic extension fields $K$, say of $k=Q$ (nor the Galois group of the algebraic closure of $Q$ ). We do not even know for a general fixed $K$ the decomposition laws explicitly which govern the prime ideal decomposition of the rational primes in $K$. We cannot truly say that we have a theory of the algebraic equations in one unknown with rational coefficients. However, in spite of all the difficulties, amazing progress has been made in Artin's work.

In Takagi's case, the Dedekind zeta-function $\zeta_{K}(s)$ of the abelian extension field of $k$ was the product of the zeta-function $\zeta_{k}(s)$ of the ground field $k$ and Dirichlet $L$-series belonging to nonprincipal characters in $k$. Hecke had shown in 1917 that the latter were entire functions. Thus, $\zeta_{k}(s)$ divides $\zeta_{K}(s)$ in the sense that the quotient is an entire function. Artin's starting point was the generalization of this problem: If $K$ is any extension field of finite degree of the algebraic number field $k$, does $\zeta_{k}(s)$ divide $\zeta_{K}(s)$ ? If this is true, there is concealed in this result information about the unknown decomposition laws for the prime ideals of $k$. Of course, it also follows that the zeros of the zeta-function of $k$ (or of the Riemann zeta-function) appear among the zeros of $\zeta_{K}(s)$.

Already in 1923, Artin could prove the divisibility in entirely new cases, for instance when $K$ is normal over $k$ with icosahedral Galois group [2]. In analyzing his method, he soon succeeded in writing the zeta-function $\zeta_{K}(s)$ of an arbitrary normal extension field $K$ of $k$ as product of $\zeta_{k}(s)$ and factors $L(s, \chi)$ formed by means of characters $\chi$ of the Galois group $G(K / k)$. It must be emphasized that the defini- 
tion of these $L(s, \chi)$ is completely different from that of the Dirichlet $L$-series. While Artin's $L$-series can be represented as infinite products extended over the prime ideals $p$ of $k$, the definition of the contribution of $p$ does not depend on the position of $p$ in an ideal class group of $k$. The key lies in the use of the Frobenius substitution $\sigma \in G(K / k)$ associated with a prime ideal divisor $\mathfrak{B}$ of $\mathfrak{p}$ in $K$. It is then clear that the form of the decomposition of $\mathfrak{p}$ in $K$ is of decisive influence. In the case of Dirichlet's $L$-series, we have congruence classes, and the behavior of $\mathfrak{p}$ with regard to these congruence classes enters into the picture. If $K$ is abelian over $k$, it is not at all clear that Takagi's and Artin's decompositions of $\zeta_{K}(s)$ coincide. In fact, this point was the most formidable obstacle in Artin's work. If one tries one of the simplest examples of quadratic extension fields $K$ of the rational field $Q=k$, one can see that the classical quadratic law of reciprocity is the bridge one has to use. In [3], Artin could settle the question in all cases where reciprocity laws for the $m$ th power residues were available. The case where $G(K / k)$ was cyclic could be treated, the case of a general $G(K / k)$ remained open.

Now one of the most fascinating developments in the history of mathematics took place. This was the realization by Artin that what was missing was a theorem of abelian class field theory, a theorem that stated that the Frobenius substitution $\sigma$ provided an explicit and canonical isomorphism of Takagi's group $\mathfrak{h} / \mathfrak{h}_{0}$ onto the Galois group $G(K / k)$. He saw clearly that it was this theorem which was the key to the laws of reciprocity in number theory. Hermann Weyl, in another context, has spoken of the "Blitzschlag des Gedankens, der den Holzstoss der Formeln in Brand setzt." This seems to describe perfectly what must have happened in Artin's mind.

In 1923, no possibility of proof for this new theorem was in sight. In reading [3], one has the feeling that though Artin then did not know how to approach his conjecture, he had full confidence that some day, sooner or later, he would have a proof. I wonder if this confidence in his own powers in a young man caused raised eyebrows among some of the older mathematicians of those days. If so, they knew better a few years later.

There had been an important development. Tschebotareff in 1925 had succeeded in proving a conjecture of Frobenius about the density of the set of prime ideals of a normal extension field $K$ for which the Frobenius substitution lies in a given conjugate class of $G(K / k)$. Frobenius himself had only been able to handle certain unions of such sets. In order to separate these sets, Tschebotareff had used a process 
of "crossing" the field with cyclotomic fields. Artin recognized that this idea could be used in his case.

Now, the situation could be reversed. [5] contains a beautiful direct proof of what is now known as Artin's law of reciprocity. It is no longer necessary to apply the older reciprocity laws for the proof. In fact, they are now obtained as easy corollaries of the new theorem. The significance of Artin's theorem goes even beyond that. It is not only a supplement to the abelian class field theory; it has become the central result.

It is well known that Gauss again and again took up the question of the classical quadratic law of reciprocity, never satisfied with the existing proofs and always giving new ones. In a certain sense, the classical theorem appeared to have been given its final form by Artin in 1927.

All the results of [3] were now firmly established. In particular, it was now shown that Artin's new $L$-series could be continued analytically over the whole plane and that they satisfied functional equations of the types known from the zeta-functions and the Dirichlet $L$-series. There were important points to be cleared up. [8] and its companion paper [9] contain a new systematic treatment of the $L$ series. In [3], an analytic trick had been used in order to avoid the discussion of the ramified primes. Tricks could not satisfy Artin for any length of time. He now faced the difficulty in what one might call a frontal attack. This led to an important new insight into the nature of this question.

A particular application of Artin's law of reciprocity must be mentioned. One of Hilbert's conjectures on absolute class fields had remained open; in fact, it appeared particularly mysterious. This is the "Principal Ideal Theorem." Now, Artin could show that it would follow from a property of finite metabelian groups. This group theoretical statement was first proved by Furtwängler in 1930. A simple proof was given by Iyanaga in 1934, then a member of Artin's circle in Hamburg.

We may come back once more to the question of divisibility of zetafunctions from which we started. In the case of an arbitrary extension field $K$, it is still open. In the case of a normal extension field $K$ of $k$, Artin's methods were strong enough to provide a proof. All that was needed was an easy group theoretical lemma. This lemma was proved by Aramata in 1933. There is no doubt that Artin could have done this easily, if he had ever tried. The reason that he did not try was typical for him. His attention in the mean time had become focussed 
on a much bigger question. His new conjecture was that all his $L$ functions belonging to irreducible nonprincipal characters were entire functions. This would imply the divisibility of zeta-functions in the general case, but would still be much deeper.

Artin's conjecture is still open. It has taken its place beside the Riemann conjecture for the Dedekind zeta-functions as one of the great open problems of algebraic number theory.

Artin's work on which we have reported here has its roots in abelian class field theory. A reader who may want to see more clearly its general impact and its significance for a "nonabelian" theory should consult Hasse's report. ${ }^{3}$

Finally, two joint papers of Artin and Hasse [4], [6] must be mentioned. They deal with the supplementary theorems for the reciprocity theorems for power residues. They are perhaps closer to Hasse's sphere of interest than to Artin's.

We shall return to class field theory again and again, but we will now first discuss the other principal results obtained by Artin during the years 1921-1931. One of his most outstanding achievements washis solution of Hilbert's Problem XVII which had withstood all previous attempts. The question which had arisen in Hilbert's investigations on the foundations of geometry was this: Given a rational function $F=F\left(x_{1}, x_{2}, \cdots, x_{n}\right)$ of $n$ variables with rational coefficients such that $F$ does not take negative values for real values of the variables. Can $F$ be written as a sum of squares of rational functions with rational coefficients? In [20], Artin answered this in the affirmative obtaining at the same time generalizations and refinements. His method was as remarkable as the result. It was perhaps the first triumph of what is sometimes called "abstract" algebra. The basis was formed by the theory of formally real fields developed by Artin and Schreier ${ }^{4}$ in [19] and [21].

It is the aim of this theory to characterize by purely algebraic properties those fields for which the familiar theorems on zeros of polynomials with real coefficients hold. A field $K$ is "real" in the sense of Artin and Schreier, if in $K$ the element -1 cannot be represented as a sum of squares. Of course, this property is preserved under field

3 H. Hasse, Bericht uber neuere Untersuchungen und Probleme aus der Theorie der algebraischen Zahlkörper, Part II. Ergänzungsband 6 des Jahresbericht der Deutschen Mathematiker Vereinigung. Leipzig und Berlin 1930.

4 Otto Schreier, whose work is well remembered in a number of connections, had been a student of Furtwängler in Vienna. He then became Privatdozent in Hamburg. He died in 1929 at the age of 28, shortly after having been appointed to a professorship in Rostock. 
isomorphism. It is also clear that every ordered field is "real." A field $P$ is "real-closed," if it is "real" while no proper algebraic extension field is. It was shown that a "real-closed" field $P$ could be ordered in a unique way, and that then in $P$ the familiar theorems of real algebra in the usual sense held. For instance, if $f(x)$ is a polynomial with coefficients in $P$ and if $f(a)<0, f(b)>0$ with $a, b \in P$, there exist zeros $c \in P$ of $f(x)$ between $a$ and $b$. "Real-closed" fields can be characterized as fields which are not algebraically closed, but possess simple algebraically closed extensions. In fact, it suffices to adjoin a square root of -1 to a "real-closed" field in order to obtain an algebraic closure. Conversely, every algebraically closed field $\Omega$ of characteristic 0 contains "real-closed" fields $P$ with $\Omega=P\left((-1)^{1 / 2}\right)$. For arbitrary "real" fields $K$, it is shown that there exist algebraic, "realclosed" extension fields, and hence orderings of $K$. If $K$ is an ordered field, there exists an essentially unique algebraic, "real-closed" extension whose ordering extends that of $K$.

We next turn to Artin's Ph.D. Thesis [1]. It is clear from the description of abelian class field theory that there may exist other fields $k$ besides algebraic number fields for which a similar theory can be developed. This is indeed the case for fields $k$ of algebraic functions of one variable $t$ over a field of constants $\Gamma$ with a finite number of elements. Artin in his dissertation considers the case of a quadratic extension field of the field of rational functions of $t$; the field $\Gamma$ is taken as the prime field. He was specially interested in the corresponding zeta-function, and he could verify here the Riemann conjecture in a number of special cases. Much of this work shows already the future master. It discloses a very great skill with computations, a feature one might not have guessed.

The thesis was the first step in an important development. F. K. Schmidt a little later considered the general case of function fields of transcendence degree 1 over finite fields and discovered the functional equation of the zeta-function and its connection with the Riemann-Roch theorem. Then Hasse proved the Riemann conjecture for function fields of genus 1 . In the 1940's, Weil finally succeeded in proving both the Riemann conjecture for function fields of arbitrary genus and the Artin conjecture on $L$-series. This superb achievement convinced Artin of the necessity for an axiomatic treatment of class field theory joining the two cases. It was his hope that by understanding properly the common element of both theories and by analyzing Weil's ideas, one might find the way to prove the two conjectures in the number field case too. Only the future can show if he was right. 
We next mention briefly the two papers [24] and [25] on hypercomplex numbers, published in 1928. In the first, Artin extended Wedderburn's theory of algebras to noncommutative rings with chain conditions. This is the reason that the name "Artin ring" is now used for rings with minimum condition for one-sided ideals. In the second paper, Artin studied the arithmetic in a semisimple algebra over the field of rational numbers giving not only a simpler approach to a theory developed by Speiser, but also extending the work much further.

It seems that right from the beginning, Artin was aware that some kind of connection existed with class field theory. While some reasons for this could be given, the nature of this connection was not obvious, and one can only marvel at Artin's insight.

Artin's work leads naturally to an analytic number theory of hypercomplex numbers and to the introduction of a zeta-function. This was the topic of the Ph.D. Thesis of Käthe Hey written under Artin's direction. It was Zorn, also a student of Artin, who showed that these results could be applied in class field theory.

The next progress in this connection came from another side, from Hasse. Hasse first used hypercomplex arithmetic to give a proof of Artin's law of reciprocity. In a way, this was the first version of what was to become the use of cohomological methods in class field theory.

A paper [34] of Artin in 1925 dealt with the theory of braids, a topic very much Artin's own. Its special fascination lies in the interrelation of topological and group-theoretical questions. Artin was to return to this field in 1946, [36], [37]. He himself has given a nontechnical report [39] which everybody interested in Artin's work should read. Nobody could match Artin's skill and power of presentation, and we shall not try it.

Finally there are a number of shorter papers, a topological paper [35] dealing with two-dimensional surfaces in a four-dimensional Euclidean space, a paper [40] on mechanical systems with quasiergodic orbits (but with number theoretical undertones), a joint paper [22] with van der Waerden on chain conditions and a few other ones.

The ten year period 1921-1931 of Artin's life had seen an activity not of ten equalled in the life of a mathematician. They were followed by what may appear to a casual observer as a period of ten years of silence. This would be a false impression. It is true that Artin developed a strong aversion against the writing of papers for publication. But the essential change was that from now on, Artin spoke 
through his students and through the members of his mathematical circle. He gave his own ideas generously to his students. Some of the dissertations written under Artin were probably mostly his own work. Sometimes, he had had an idea before and led his student to find it for himself. On all of his students, ${ }^{5}$ Artin exerted a profound influence. It would be impossible to separate what was Artin's work and what was that of others and we shall not attempt this. Artin detested discussion of questions of priority. It did not matter in the least to him, whether some work was done by him or by somebody else. What mattered was that is was done the way he felt it should be done.

It was natural that the fascination of Artin's genius attracted mature mathematicians. Van der Waerden, Iyanaga, Herbrand, and Chevalley came to work in Hamburg. Zassenhaus and Witt were members of the Hamburg Mathematics Department. All these men and many others later have acknowledged freely the influence Artin had on them.

Many of the publications during the later part of Artin's life represented joint work. The paper [26] on the sum of two sets of integers by Artin and Scherk was included by Khintchine in his book "Three pearls of the theory of numbers." It deals with a simplification of H. B. Mann's proof of the so-called $(\alpha, \beta)$-hypothesis. There were three joint papers of Artin and Whaples; [27] deals with the theory of simple rings, and [12] and [13] with an axiomatic characterization of fields by the product formula for valuations. The fields in question are the two types of fields $k$ mentioned above in connection with class field theory. Together with Ankeny and Chowla, Artin published two papers on the class numbers of real quadratic fields [15], [16]. A paper of Artin and R. H. Fox [38] contains some ingenious examples of subsets of a spherical $n$-dimensional space $S^{n}$ which are homeomorphic images of Euclidean polyhedra, but are wildly imbedded in $S^{n}$. In [31], Artin and Tate proved a number of results on extensions of Noetherian rings. From one of them, a theorem of Zariski can be deduced, which Zariski had used for a short and elegant proof of Hilbert's Nullstellensatz.

Throughout his teaching career, Artin had given courses on class field theory. Their form had changed radically, since each new progress had been incorporated. In particular, Chevalley's fundamental paper in 1940 had caused a complete revision. While each version of Artin's lectures could be said to be of permanent interest, it seems

- A list of Artin's students and of the titles of their dissertations can be found in Zassenhaus' article. 
that only in the nineteen fifties, he began to think seriously of writing a book on class field theory.

Cohomological algebra had then been developed on a broad basis by H. Cartan, Eilenberg and MacLane. The importance of cohomological methods for class field theory had become increasingly clear, for instance in the work of Hochschild. For the first time, Artin seemed to have felt that he could present the theory in a form which really satisfied him. A large fragment of what was meant to be a first manuscript, consisting of somewhat revised notes of a 1951-1952 seminar, was finally published at Harvard University in 1961 in lecture note form under the title Class field theory by Artin and Tate. A large part of these notes dealt with new results and could be considered as a series of research papers. In particular, we mention an abstract generalization of class field theory, the theory of class formations, and the great advances in the cohomological theory made by Tate soon after the seminar. Artin's law of reciprocity can now be viewed as a special case of the theorem of Tate dealing with the higher cohomology groups.

There are a number of other books and sets of lecture notes by Artin. Each of them presents a novel approach. There are always new ideas and new results. It was a compulsion for Artin to present each argument in its purest form, to replace computation by conceptual arguments, to strip the theory of unnecessary ballast. What was the decisive point for him was to show the beauty of the subject to the reader. He himself has said [46]: "We all believe that mathematics is an art. The author of a book, the lecturer in a classroom tries to convey the structural beauty of mathematics to his readers, to his listeners. In this attempt, he must always fail. Mathematics is logical to be sure, each conclusion is drawn from previously derived statements. Yet the whole of it, the real piece of art, is not linear; worse than that, its perception should be instantaneous. We all have experienced on some rare occasions the feeling of elation in realizing that we have enabled our listeners to see at a moment's glance the whole architecture and all its ramifications."

Much of the contents of Artin's books and lecture notes have become standard materials in courses. In particular, the books on Galois theory, algebraic number theory (in various forms) and geometric algebra are especially well known. The notes [42] and [43] may be considered as a chapter of a book on complex variables. We should also mention Artin's Einfïhrung in die Theorie der GammaFunktion (Hamburger Mathematische Einzelschriften, 1931), and the Rings with minimum condition by Artin, Nesbitt and Thrall (Univer- 
sity of Michigan Press, 1944). A list of Artin's books and lecture notes given in the collected papers is reproduced below. There is one notable addition, a set of notes on algebraic topology by Artin and Hel Braun on which the two authors had collaborated during the last years of his life. ${ }^{6}$

The lines of Artin quoted above show the importance of teaching for Artin. Each of his courses was carefully planned; the smallest detail received his full attention. There was a strict economy with the aim to give a balanced picture of a subject in a limited time. No proof which was necessary was ever omitted or only sketched; this was not mathematics for him. There was the same attitude in seminars too. Students would never get away with incomplete arguments. There was no haste in seminars. Topics were only left when they had been explored fully. The final effect was that of a recreation of a theory rather than that of a presentation. Artin never really liked much to attend colloquium talks. Perhaps the reason was the different attitude the speaker is usually forced to take.

There is the saying of G. B. Shaw about people who do things and people who teach things. If Shaw had ever met Artin, he might have grasped that there were men who were creative when they taught. Of course, Shaw could have discovered this by reading about Socrates. ${ }^{7}$ In any case, it was of the highest importance to Artin to help his students to develop their own mathematical personalities, to assist them to stand on their own feet, to kindle in them a deep love of mathematics. By putting all his strength to this effort and by using all his ingenuity, he created, not mathematical theories, but mathematicians.

A few words should be said about some of the problems left open by Artin. Nonabelian class field theory and the $L$-series have already been discussed. Some of the unsolved problems on braids are mentioned in the introduction of [36]. The whole theory of braids was only to be a beginning. Much more general questions of topology were to be studied. Artin's papers on finite simple groups [32], [33] were meant more to disclose problems than to answer them. At some time in the future, the group theorists will have to come to grips with them.

- Among the books in which the authors mention explicitly their indebtedness to Artin are: B. L. van der Waerden, Moderne Algebra; H. Zassenhaus, Lehrbuch der Gruppentheorie; Hel Braun und Max Koecher, Jordan-Algebren.

7 The same reading is also to be recommended to people who still measure achievement by the number of pages of publications. 
Some conjectures of Artin are discussed by Lang and Tate in the preface of the collected papers of Artin. One conjecture deals with the density of the set of primes for which a given integer is a primitive root. Perhaps the idea may look deceptively simple. No answer is in sight.

Another conjecture states that if $f$ is a homogeneous polynomial of degree $d$ in $n$ variables with coefficients in a $p$-adic field $Q_{p}$ and if $n>d^{2}$, then $f$ has a nontrivial zero in $Q_{p}$. This has been proved in 1965 by $\mathrm{Ax}$ and Kochen, provided that, for given $d$, a finite set $A(d)$ of primes $p$ depending on $d$ is excluded. Very recently, Terjanian has shown that there exist counterexamples, if all primes $p$ are admitted. There are also corresponding global conjectures of Artin. They will now have to be modified somewhat. No progress has been made on them.

A conjecture of Furtwängler might be mentioned in which Artin had always been interested, the class field tower problem. He had remarked that if Minkowski's inequalities for the discriminants of algebraic number fields could be improved, an affirmative answer could be given. ${ }^{8}$ I. R. Safarevič and E. S. Golod have shown in 1964 that actually the answer to the problem is negative, that infinite towers of class fields exist. This makes Artin's remark no less interesting, since one can now use it in the opposite direction.

I cannot help feeling regret that Artin did not live to see these last developments. He would have been enthusiastic about the work on these two conjectures.

Artin's very nature destined him to have a profound influence on the mathematics of our times. This is not the place to try to evaluate the importance of Artin's ideas for the work of other mathematicians. As an indication that his impact is not going to diminish, we may take the remarks of H. Cartan on the influence of Artin on Bourbaki.

For Artin, to be a mathematician meant to participate in a great common effort, to continue work begun thousands of years ago, to shed new light on old discoveries, to seek new ways to prepare the developments of the future. Whatever standards we use, he was a great mathematician.

\section{BIBLIOGRAPHY}

The collected papers of Artin edited by S. Lang and John T. Tate have been published by Addison-Wesley.

$8 \mathrm{cf}$. Hasse, Bericht Teil. I, Jahresbericht der Deutschen Mathematiker Vereinigung 35 (1926) 46. 


\section{THESIS}

1. Quadratische Körper im Gebiet der höheren Kongruenzen. I, II, Math. Z. 19 (1924), 153-246.

\section{Class FIELd THEORY}

2. Über die Zetafunktionen gewisser algebraischer Zahlkörper, Math. Ann. 89 (1923), 147-156.

3. Über eine neue Art von L-Reihen, Hamb. Abh. 3 (1923), 89-108.

4. Über den zweiten Ergänzungssatz zum Reziprozitätsgesetz der l-ten Potenzreste im Körper $k_{\zeta}$ der l-ten Einheitswurzeln und in Oberkörpern von $k_{\zeta}$ (with H. Hasse), J. Reine Angew. Math. (1925), 143-148.

5. Beweis des allgemeinen Reziprozitätsgesetzes, Hamb. Abh. 5 (1927), 353-363.

6. Die beiden Ergänzungssätze zum Reziprozitätsgesetz der ${ }^{n}{ }^{n}$-ten Potenzreste im Körper der ${ }^{n}$-ten Einheitswurzeln (with H. Hasse), Hamb. Abh. 6 (1928), 146-162.

7. Idealklassen in Oberkörpern und allgemeines Reziprozitätsgesetz, Hamb. Abh. 7 (1929), 46-51.

8. Zur Theorie der L-Reihen mit allgemeinen Gruppencharakteren, Hamb. Abh. 8 (1930), 292-306.

9. Die gruppentheoretische Struktur der Diskriminanten algebraischer Zahlkörper, J. Reine Angew. Math. 164 (1931), 1-11.

\section{ALgEBRAIC NUMBER THEORY}

10. Über Einheiten relativ galoisscher Zahlkörper, J. Reine Angew. Math (1932), 153-156.

11. Über die Bewertungen algebraischer Zahlkörper, J. Reine Angew. Math. (1932), 157-159.

12. Axiomatic characterization of fields by the product formula for valuations (with G. Whaples), Bull. Amer. Math. Soc. 51 (1945), 469-492.

13. A note on axiomatic characterization of fields (with G. Whaples), Bull. Amer. Math. Soc. 52 (1946), 245-247.

14. Questions de base minimale dans la théorie des nombres algébriques, Colloques Internationaux du CNRS No. 24, Paris, 1950, pp. 19-20.

15. The class-number of real quadratic fields (with N. C. Ankeny and S. Chowla), Proc. Nat. Acad. Sci. U.S.A. 37 (1951), 524-525.

16. The class-number of real quadratic number fields (with N. C. Ankeny and S. Chowla), Ann. of Math. 56 (1952), 479-493.

17. Representatives of the connected component of the idele class group, Proc. Internat. Sympos. Algebraic Number Theory, Tokyo \& Nikko, 1955, pp. 51-54.

\section{REAL FIELDS}

18. Kennzeichnung des Körpers der reellen algebraischen Zahlen, Hamb. Abh. 3 (1924), 319-323.

19. Algebraische Konstruktion reeller Körper (with O Schreier), Hamb. Abh. 5 (1926), 85-99.

20. Über die Zerlegung definiter Funktionen in Quadrate, Hamb. Abh. 5 (1927), 100-115.

21. Eine Kennzeichnung der reell abgeschlossenen Körper (with O. Schreier), Hamb. Abh. 5 (1927), 225-231. 


\section{ALgebra AND NUMBER THEORY}

22. Die Erhaltung der Kettensätze der Idealiheorie bei beliebigen endlichen Körpererweiterungen (with B. L. van der Waerden), Nachr. Akad. Wiss. Göttingen Math. Phys. Kl. II (1926), 23-27.

23. Über einen Satz von Herrn J. H. Maclagan Wedderburn, Hamb. Abh. 5 (1928), 245-250.

24. Zur Theorie der hyperkomplexen Zahlen, Hamb. Abh. 5 (1928), 251-260.

25. Zur Arilhmetik hyperkomplexer Zahlen, Hamb. Abh. 5 (1928), 261-289.

26. On the sums of two sets of integers (with P. Scherk), Ann. of Math. 44 (1943), 138-142.

27. The theory of simple rings (with G. Whaples), Amer. J. Math. 65 (1943), 87-107.

28. The free product of groups, Amer. J. Math. 69 (1947), 1-4.

29. Linear mappings and the existence of a normal basis, Studies and Essays Presented to R. Courant on his 60th Birthday, Interscience, New York, 1948, pp. 1-5.

30. Remarques concernant la theorie de Galois, Colloques Internationaux du CNRS, No. 24, Paris, 1950, pp. 161-162.

31. A note on finite ring extensions (with J. T. Tate), J. Math. Soc. Japan 3 (1951), 74-77.

32. The orders of the linear groups, Comm. Pure Appl. Math. 8 (1955), 355-365.

33. The orders of the classical simple groups, Comm. Pure Appl. Math. 8 (1955), $455-472$.

\section{Topology}

34. Theorie der Zöpfe, Hamb. Abh. 4 (1925), 47-72.

35. Zur Isotopie zzeeidimensionaler Flächen im $R_{4}$, Hamb. Abh. 4 (1925), 174-177.

36. Theory of braids, Ann. of Math. 48 (1947), 101-126.

37. Braids and permutations, Ann. of Math. 48 (1947), 643-649.

38. Some wild cells and spheres in three-dimensional space (with R. H. Fox), Ann. of Math. 49 (1948), 979-999.

39. The theory of braids, American Scientist 38 (1950), 112-119.

\section{Miscellaneous}

40. Ein mechanisches System mit quasiergodischen Bahnen, Abh. Math. Sem. Univ. Hamburg 3 (1924), 170-175.

41. Coordinates in affine geometry, Notre Dame Math. Colloquium, 1940, pp. 15-20.

42. On the independence of line integrals on the path, Proc. Nat. Acad. Sci. U.S.A. pp. 27 (1941), 489-490. $55-70$.

43. On the theory of complex functions, Notre Dame Math. Lectures No. 4, 1944,

44. A proof of the Krein-Milman theorem, Picayune Sentinel, University of Indiana, 1950 (a letter to M. Zorn).

\section{General}

45. The influence of $J . H . M$. Wedderburn on the development of modern algebra, Bull. Amer. Math. Soc. 56 (1950), 65-72.

46. Review of Bourbaki's Algebra, Bull. Amer. Math. Soc. 59 (1953), 474-479. 5-12.

47. Contents and methods of an algebra course, Tata Institute, Bombay, 1960, 
48. Die Bedeutung Hilberts filr die moderne Mathematik, Jahrbuch Akad. Wiss. Göttingen, 1962 (address given for Hilbert's 100th birthday).

49. Zur Problemlage der Mathematik (lecture broadcast from RIAS).

\section{BOOKS AND LECTURE NOTES}

50. Einfïhrung in die Theorie der Gammafunktion, Hamburger mathematische Einzelschriften 1. Heft, 1931.

51. Galois Theory, Notre Dame Math. Lectures No. 2, 1942, 82 pp.

52. Galoische Theorie, Leipzig, 1959; (a translation and expansion of the preceding item).

53. Modern higher algebra, Galois theory, (Notes by A. Blank) Lectures, New York University, 1947.

54. Rings with minimum condition (with C. J. Nesbitt and R. M. Thrall), Michigan, 1948.

55. Algebraic numbers and algebraic functions, (Notes by I. Adamson), New York University, 1950.

56. Modern developments in algebra, (Notes by R. Stoll), Boulder summer conference, 1953.

57. Selected topics in modern algebra, Summer conference at University of North Carolina, 1954.

58. Elements of algebraic geometry, (Notes by G. Bachman), New York University, 1955.

59. Calculus and analytic geometry, (Notes by G. B. Seligman), Princeton 1957.

60. Algebraic number theory, (Notes by G. Wurges), Göttingen, 1956.

61. Geometric algebra, Interscience, New York, 1957.

62. Class field theory (with J. T. Tate), Harvard, 1961.

63. Emil Artin and Hel Braun, Vorlesungen über algebraische Topologie, (Notes by A. Thedy), Mathematisches Seminar, Universität Hamburg.

\section{REFERENCES}

H. Cartan, Emil Artin, Abh. Math. Sem. Univ. Hamburg 28 (1965), 1-6.

C. Chevalley, Emil Artin, Bull. Soc. Math. France 92 (1964), 1-10.

H. Zassenhaus, Emil Artin, his life and his work, Notre Dame J. Formal Logic 5 (1964), 1-9. 